\title{
X-RAY ABSORPTION AND EXPERIMENTS WITH CIRCULARLY POLARIZED RADIATION
}

\author{
B. SChMiedeskamp
}

Fakultät für Physik, Universität Bielefeld, 33615 Bielefeld, Germany

\begin{abstract}
Absorption of circularly polarized radiation ( $\sigma$-radiation) recently gained interest mainly due to its helicity-dependence for magnetic materials (circular magnetic dichroism). The physics behind this absorption process is optical orientation and is closely related to the physics behind other types of experiment with circularly polarized radiation in which fundamental electronic properties of matter are studied. The consideration of X-ray absorption plays also a role in the development of new beam lines for $\sigma$-radiation. An important part in a beamline for $\sigma$-radiation in the soft X-ray range can be multilayer optics. The role of absorption in the design of these optics is discussed as well as different types of experiment with circularly polarized radiation: the physical mechanism of optical orientation is applied to gain insight into both magnetic and fundamental electronic properties of matter.
\end{abstract}

PACS numbers: $75.25 .+z, 41.60 . \mathrm{Ap}$

\section{Introduction}

We start with a discussion of the propagation of an electromagnetic wave in matter which can be characterized by means of optical constants. Its imaginary part describes absorption. Absorption is already an important factor in the design of monochromator beamlines, because it limits the maximum reflectivities of optical components achievable and finally results in thermal loads on these components. The role of absorption in multilayer soft X-ray mirrors is discussed.

The second part deals with circularly polarized radiation (CPR). Different sources of CPR are briefly described. Then different types of experiment with CPR are discussed. The absorption process is then regarded as a process in which a photon is annihilated, resulting in an excited state of matter and/or emission of electrons. 


\section{Propagation of an electromagnetic wave in matter, optical constants}

When an electromagnetic wave propagates through a material, its intensity is exponentially attenuated [1]:

$$
I=I_{0} \exp (-\alpha z)
$$

with $z$ - the thickness in the material, $\alpha$ - the linear attenuation coefficient (also absorption coefficient) and $I_{0}$ - the intensity at $z=0$. The propagation of the wave can be described by [1]:

$$
E=E_{0} \exp \left(-\frac{2 \pi \beta z}{\lambda}\right) \exp \left[-\frac{2 \pi \mathrm{i}}{\lambda}(n z-c t)\right]
$$

with $E_{0}$ - the amplitude of the field at $z=0, \lambda$ - the wavelength in vacuum and $c$ - the speed of light. $\tilde{n}=n+\mathrm{i} \beta$ is the complex.refractive index of the material. Its imaginary part, $\mathrm{i} \beta$ describes the absorption of the wave in matter. In some works $\beta$ is denoted as absorption coefficient, $\alpha$ is then given by $\alpha=4 \pi \beta / \lambda$.

In the $\mathrm{X}$-ray region photon energies are large compared to binding energies of valence electrons. It is then useful to describe the optical properties of matter by means of atomic scattering factors $f=f_{1}+\mathrm{i} f_{2} . \tilde{n}$ is then given by [1]:

$$
\tilde{n}=1-\delta+\mathrm{i} \beta=1-\frac{r_{0} \lambda^{2}}{2 \pi} N_{\text {at }}\left(f_{1}+\mathrm{i} f_{2}\right)
$$

The values of $f_{1}$ and $f_{2}$ are tabulated by Henke et al. [2] for photon energies between $10 \mathrm{eV}$ and $10 \mathrm{keV}$. The optical constants $f_{1}$ and $f_{2}$ are deduced from absorption measurements and are believed to be accurate between $100 \mathrm{eV}$ and $10 \mathrm{keV}$ with the exception of energies close to absorption edges where fine structure is produced by the interaction with neighbouring atoms. At photon energies far below $100 \mathrm{eV}, f_{1}$ and $f_{2}$ depend also on the chemical bonding between the atoms. Figure 1 shows values of $f_{1}$ and $f_{2}$ between $100 \mathrm{eV}$ and $2 \mathrm{keV}$ vs. atomic number $Z$ of the atoms. Low values of $f_{2}$ are obtained at the low energy side of absorption edges. In the limit of very high energies $(h \nu \gg$ binding energy of electrons in the $k$ shell), $f_{1}$ approaches $Z$ and $f_{2}$ vanishes.

When an electromagnetic wave traverses an interface between two materials with refractive indices $\tilde{n}_{a}$ and $\tilde{n}_{b}$ the wave is partly reflected. The reflection coefficient $r_{a b}=E_{\mathrm{r}} / E$ ( $E_{\mathrm{r}}$ - reflected amplitude) of the wave can be calculated by means of the Fresnel equations [3]. The reflectivity $R_{a b}$ is $R_{a b}=\left|r_{a b}\right|^{2}$.

For normal incidence and abrupt interfaces $r_{a b}$ is given by

$$
r_{a b}=\frac{\tilde{n}_{a}-\tilde{n}_{b}}{\tilde{n}_{a}+\tilde{n}_{b}} .
$$

For low photon energies up to $\approx 20 \mathrm{eV}$ materials exist with $\beta \approx 1$ [2], resulting in reflectivities of roughly $25 \%$ for an interface with vacuum

$$
\left(R=\left|\frac{-\delta+\mathrm{i} \beta}{2-\delta+\mathrm{i} \beta}\right|^{2} \approx \frac{\beta^{2}}{4}\right) .
$$

This is the basis for normal incidence optics in many synchrotron radiation beamlines for XUV radiation. 

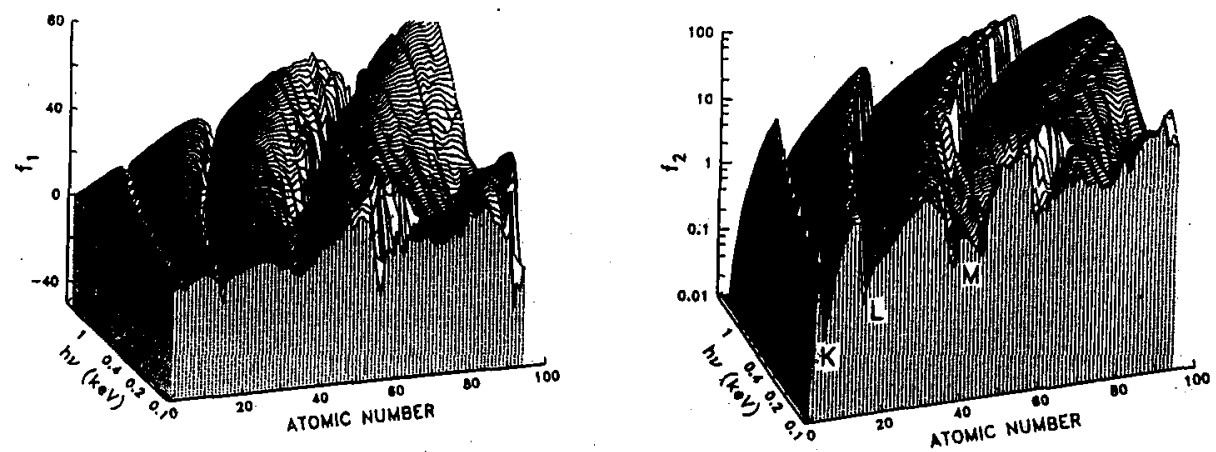

Fig. 1. Atomic scattering factors $f_{1}$ and $f_{2}$ vs. atomic number and photon energy (data from Ref. [2]).

At higher photon energies both $\delta$ and $\beta$ are considerably smaller than 1 and $R_{a b}$ is approximately given by

$$
R_{a b}=\frac{\left(\delta_{b}-\delta_{a}\right)^{2}+\left(\beta_{a}-\beta_{b}\right)^{2}}{4}
$$

$R_{a b}$ scales thus (see Eq. (2)) roughly with $\lambda^{4}$ and normal incidence reflectivities from single interfaces are thus extremely small in the soft and hard X-ray region. High reflectivities can, however, be obtained at grazing incidence, because $n \leq 1$ for almost all materials and photon energies. Total reflection occurs therefore at grazing incidence angles smaller than a critical angle $\Theta_{\mathrm{c}}$ with

$$
\sin \Theta_{\mathrm{c}} \approx \sqrt{2 \delta}
$$

(absorption losses are neglected in this estimation).

This is the basis for grazing incidence optics in many beamlines for soft X-rays.

\section{Multilayer soft X-ray optics}

By means of multilayers [4] normal incidence optics can be extended to the soft $\mathrm{X}$-ray region $(50 \mathrm{eV}$ to approximately $500 \mathrm{eV}$ ). The principle is identical to that of a quarter wave stack in the visible. The multilayers consist of alternately deposited layers of two materials $a$ and $b$. For normal incidence of the radiation one obtains constructive interference of the contributions from different interfaces if the thicknesses of the layers $d_{a}$ and $d_{b}$ are about a quarter wavelength $\lambda / 4$. Materials $a$ and $b$ must be chosen in a way that $\left|r_{a b}\right|$ is a maximum and absorption losses in the stack are minimized. Large normal incidence reflectivities can be expected if

$$
1 / \alpha \gg N\left(d_{a}+d_{b}\right) \text {. }
$$

$1 / \alpha$ - attenuation length of the wave in the multilayer stack. $N$ - number of layer pairs which is required to obtain $\frac{N}{2}\left|r_{a b}\right| \approx 1$. All materials in the soft X-ray range are strong absorbers. Nevertheless, the condition (6) is usually fulfilled if for at least one of the materials $a$ and $b$ the wavelength is on the low energy side of 
an absorption edge of a light material and the second material is chosen in order to maximize $\left|r_{a b}\right|$.

A good choice for photon energies between 50 and $100 \mathrm{eV}$ is $\mathrm{Mo} / \mathrm{Si}$. Si has a low absorption due to the Si $L$-edge at $100 \mathrm{eV}$. Mo results in high values of $\left|r_{a b}\right|$, which in this case is due to a large $\delta$ and not a large $\beta$ value. This
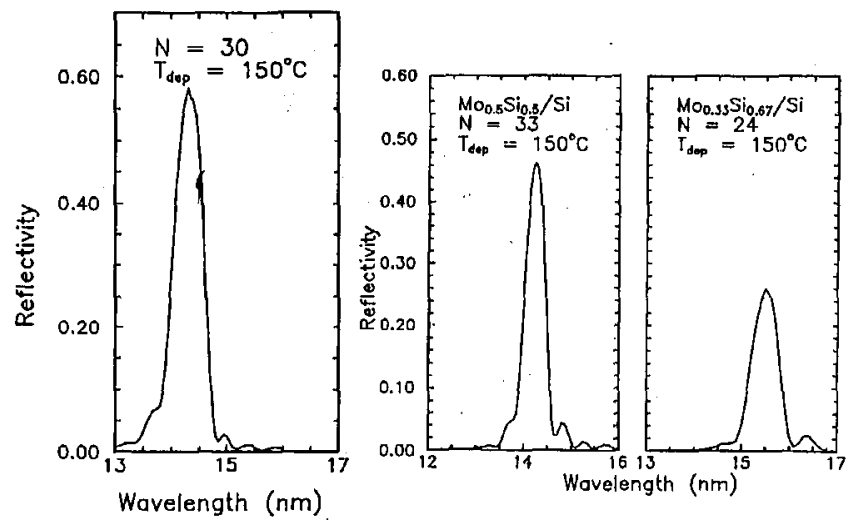

Fig. 2. Normal incidence soft X-ray reflectivities for Mo/Si- (left), $\mathrm{Mo}_{0.5} \mathrm{Si}_{0.5} / \mathrm{Si}$ - (middle) and $\mathrm{Mo}_{0.33} \mathrm{Si}_{0.66} / \mathrm{Si}$ - (right) multilayers [48].

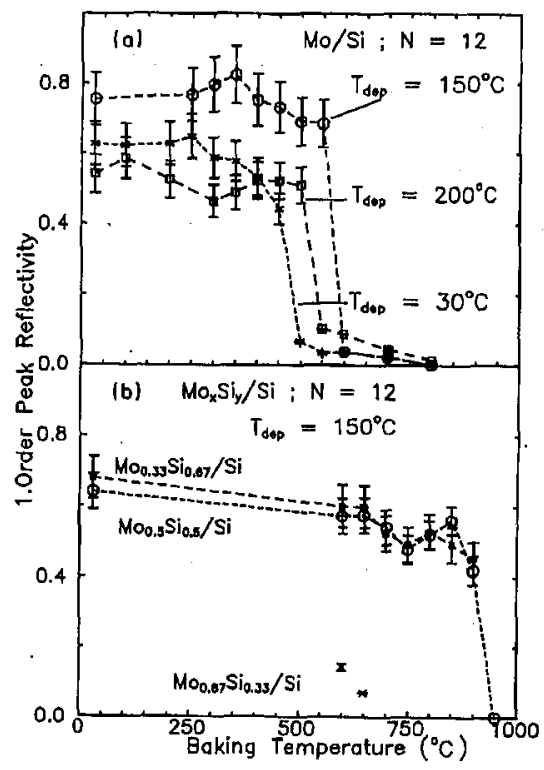

Fig. 3. Reflectivities of $\mathrm{Cu} K_{\alpha}$ radiation vs. baking temperature [48]: (a) Mo/Si multilayers deposited at different temperatures $T_{\mathrm{dep}}$, (b) $\mathrm{Mo}_{x} \mathrm{Si}_{y} / \mathrm{Si}$ multilayers with different portions $x$ and $y$ of Mo and $\mathrm{Si}$ in the absorber layers. 

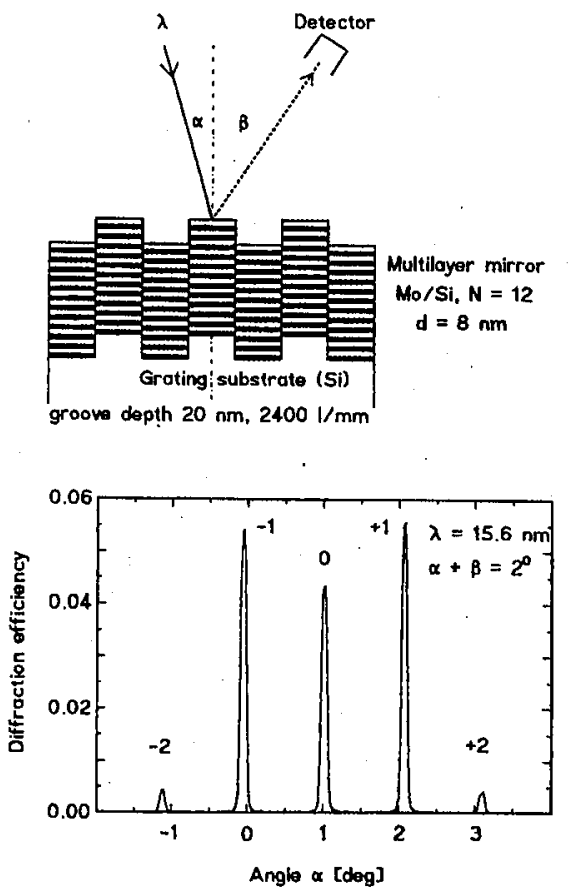

Fig. 4. Top: multilayer coated diffraction grating and the geometry for its soft X-ray optical characterization [48]. Bottom: diffraction efficiencies of the -2 nd to +2 nd diffraction orders at $\lambda=15.6 \mathrm{~nm}$. The multilayer diffraction grating has been rocked with respect to the incoming beam while leaving the detector fixed (i.e. $\alpha+\beta=$ constant $=$ $2 \mathrm{deg}$ ). The numbers at the peaks denote the diffraction orders.

keeps absorption losses in the stack very low. Figure 2 shows normal incidence reflectivities of $\mathrm{Mo} / \mathrm{Si}$ multilayers. Values around $60 \%$ can be achieved. In order to obtain a higher thermal stability of the systems against interdiffusion one can deposit multilayers with mixed $\mathrm{Mo}_{x} \mathrm{Si}_{y}$ layers and $\mathrm{Si}$ layers. The reflectivity is then lower (Fig. 2) but - as can be seen from Fig. 3 - the stability of the stacks is higher. Deposition of a multilayer on to a grating results in an optical element which combines the high normal incidence reflectivity of a multilayer with the resolution of a grating (see Fig. 4). Such multilayer-coated gratings can be the basis for normal incidence optics in the soft X-ray region.

\section{Sources of circularly polarized radiation}

Circularly polarized radiation i.e. radiation for which the $\boldsymbol{E}$-vector describes a right or left handed screw about the propagation direction* is emitted in bending

* Circularly polarized radiation can alternatively be characterized by its handedness, helicity or photon spin. Left handed CPR has positive helicity, is also denoted as $\sigma^{+}$-radiation and its photon spin $(+\hbar)$ is parallel to the propagation direction. Right handed CPR has negative helicity $\left(\sigma^{-}\right.$-radiation) and the photon spin $(-\hbar)$ is antiparallel to the propagation direction of light. 
magnets of storage rings slightly above and below the ring planes [5], while the radiation in the ring plane is linearly polarized ( $\pi$-radiation). Though $\sigma$-radiation occurs thus very generally, experiments with circularly polarized synchrotron radiation were until recently rather the exception than the rule, because most of the monochromators do not transmit the high degree of circular polarization to the samples studied. In the XUV range few monochromators for circularly polarized synchrotron radiation work at normal incidence $[5,6]$ or use monochromators for linearly polarized light in combination with phase shifting elements. Both techniques yield almost completely polarized radiation but only for photon energies below about $35 \mathrm{eV}$. Radiation with a medium degree of circular polarization at higher photon energies up to the soft X-ray region can be preserved in the monochromization at grazing incidence $[7,8]$. Some activities exist to extend the usable photon energy range to higher values with normal incidence multilayer optics [9]. Higher degrees of circular polarization can then be expected behind a monochromator. To the knowledge of the author, this has, however, up to now not yet been verified. Phase shifting elements with multilayers used in transmission are also discussed [9]. For hard X-rays $(\approx 10 \mathrm{keV})$ single crystalline plates are used as phase retarders and yield a high degree of circular polarization [10]. Using the bending magnet radiation above (below) the storage ring plane in this energy range, a medium degree of circular polarization is also preserved with crystal monochromators $[11,12]$. Considerable effort has been spent on the development of wiggler/undulator sources for $\sigma$-radiation [13].

\section{Experiments with circularly polarized radiation, optical orientation}

When circularly polarized radiation interacts with a sample as displayed in Fig. 5 the interaction depends in general on the handedness. Experiments with circularly polarized radiation make use of these dependencies.

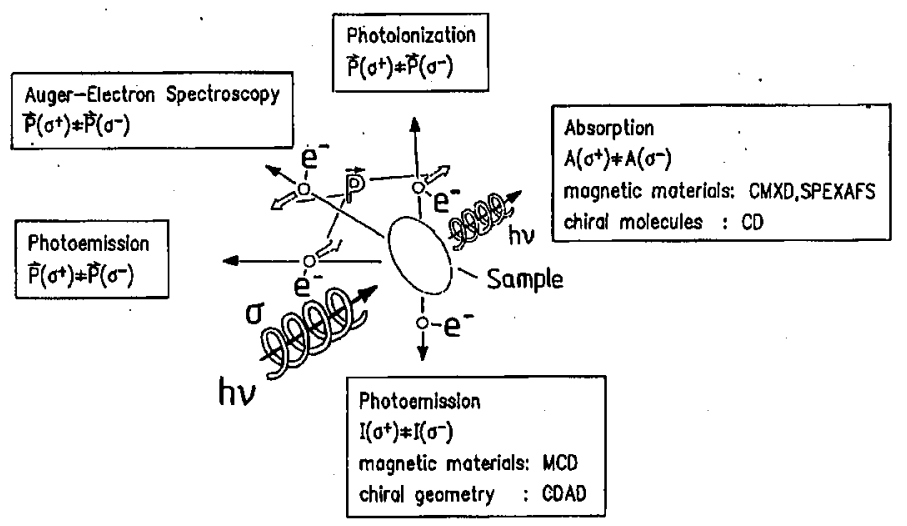

Fig. 5. The different types of experiment with circularly polarized radiation (abbreviations are explained in the text). 
The absorption $A$ and photoelectron intensity $I$ in photoemission can depend on the helicity of the radiation, if the sample is magnetic. The spin-polarization $\boldsymbol{P}$ of electrons emitted in the photoemission of free atoms and molecules, in the photoemission from solids and adsorbates as well as in an Auger process is different for left and right handed circularly polarized radiation even for non-magnetic systems.

The mechanism which produces these dependencies is optical orientation, i.e. a spin polarization transfer from the photon onto an electron. As spin polarized electrons play a key role in all cases we start with a discussion of spin polarized electrons. After that we discuss different experiments with $\sigma$-radiation: electron spin polarization (ESP) analysis in the angle- and energy resolved photoionization of free atoms and molecules [14], circular magnetic X-ray dichroism (CMXD) [11] and spin resolved extended X-ray absorption fine structure (SPEXAFS) [12] in the absorption of $\sigma$-radiation, magnetic circular dichroism (MCD) in the photoemission with $\sigma$-radiation [7], electron spin analysis in the photoemission from solids and adsorbates [15-18] and ESP analysis in the Auger electron spectroscopy from non-magnetic solids and free atoms [19].

A dependence of $A$ and $I$ can also be observed if the sample or the photoemission setup exhibits a chirality. In this case the mechanism is not optical orientation. For completeness the corresponding techniques, circular dichroism in absorption (CD) [20] and circular dichroism in the angular distribution of photoelectrons (CDAD) [21], are also given in Fig. 5 but they are not discussed further in this work.

\subsection{Spin polarized photoelectrons}

It is worth remembering that spin polarized photoelectrons can already be obtained with unpolarized radiation from unpolarized matter and that this type of spin polarization is always present in experiments with circularly polarized radiation. This has been well known from the photoionization of free atoms and molecules for more than a decade [5, 14, 22-24]. Spin-orbit interaction is required and the spin polarization is due to an interference of continuum wave functions. As illustrated in the left part of Fig. 6, symmetry considerations demand that the
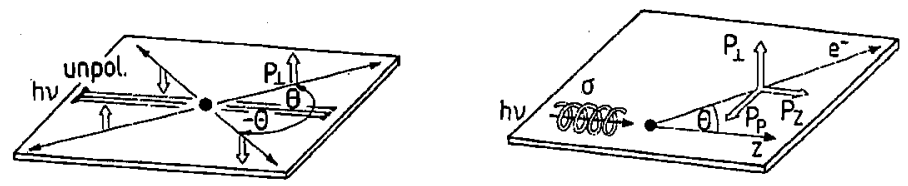

Fig. 6. Left: spin polarization in the photoionization with unpolarized radiation. Right: spin polarization in the photoionization with circularly polarized radiation.

spin polarization vector $\boldsymbol{P}$ has only a non-zero component $P_{\perp}$ perpendicular to the scattering plane of the incident radiation and the emitted electron $P_{\perp}$ is zero for $\theta=0^{\circ}, 90^{\circ}, 180^{\circ}$ and $-90^{\circ}$ and its average over all electron emission direction in space vanishes also. 
In the photoionization with circularly polarized radiation additional spin polarization is induced by optical orientation, which requires also spin-orbit interaction. This mechanism was first recognized by Fano [25] and experimentally verified by Heinzmann et al. [26]. Theoretical treatments [23] and experiments $[14,27]$ show that in this case $\boldsymbol{P}$ has in general also components $P_{\mathrm{p}}$ and $P_{\mathrm{z}}$ parallel to the reaction plane as displayed in the right part of Fig. 6 . While $P_{\perp}$ is independent of the helicity of the radiation, $P_{\mathrm{p}}$ and $P_{z}$ change their sign when the handedness of the radiation is switched from right to left handed [14, 23, 27]. Like $P_{\perp}, P_{\mathrm{p}}$ vanishes for $\theta=0,90^{\circ}, 180^{\circ}$ and $-90^{\circ}$ and its average over all electron emission directions is also zero. $P_{z}$, however, is in general non-zero for all electron emission directions and has also a non-zero average value $\bar{P}_{z}$ if all photoelectrons produced are extracted regardless of their direction of emission.

\subsection{Electron spin polarization approaching unity in the photoionization of $T l$}

Photoelectron spin polarization values close to unity are usually obtained only for special experimental conditions which require at least selection of a fixed electron emission angle $\theta$. It is an absolute exception that a beam of almost completely polarized photoelectrons occurs for all $\Theta$ simultaneously. In the photoionization of $\mathrm{Tl}$ this exception has been found even twice [28].

As displayed in the upper part of Fig. 7 the ground state of $\mathrm{Tl}$ is a ${ }^{2} P_{1 / 2}$ state. Ionization with circularly polarized radiation yields an ionic ${ }^{1} S_{0}$ state and a photoelectron which can leave as an $s_{1 / 2}$ or a $d_{3 / 2}$ continuum-wave. It can be derived that for two special combinations of the transition matrix elements $D_{s}, D_{d}$ and the phase difference $\delta_{s}-\delta_{d}$ a photoelectron spin polarization $\boldsymbol{P}$ with $|\boldsymbol{P}|=1$ can be achieved for all $\theta$ simultaneously.

The first case is shown in the lower left part of Fig. 7 . If $D_{d}=0$ the photoelectron is a pure $s$-wave resulting in $P_{z}=1$ for all $\theta$ and $\bar{P}_{z}=1$.

The second case is illustrated in the lower right part of Fig. 7. If $D_{d}=$ $-2 \sqrt{2} D_{s}$ and $\delta_{s}-\delta_{d}=0$ the outgoing $s$ - and $d$-wave interfere in such a manner that the spin polarization vector $\boldsymbol{P}$ remains in the reaction plane and is rotated by $2 \theta$ with respect to the light propagation direction for an electron emission angle $\theta$.

That these ideal cases can indeed approximately be achieved in a photoionization experiment is shown by the experimental results in the lower left and right part of Fig. 7. Both results were obtained close to autoionization resonances, where matrix elements and phases show strong variations with radiation wavelength $\lambda$. At $\lambda=148 \mathrm{~nm}$ autoionization yields a drastic increase in the cross-section $\sigma$ for photoionization, which is exclusively due to $s$-waves and results in $\bar{P}_{z}=1$ (bottom left). At $\lambda=83 \mathrm{~nm}, P_{z}$ varies with $\Theta$ as expected for case 2 (bottom right).

$\bar{P}_{z}$ can be determined [22] from the transition matrix elements $D_{s}$ and $D_{d}$ by means of

$$
\bar{P}_{z}=\frac{D_{s}^{2}-0.5 D_{d}^{2}}{D_{s}^{2}+D_{d}^{2}} .
$$

As expected we obtain $\bar{P}_{z}=1$ for case 1 . Case 2 yields $\bar{P}_{z}=-\frac{1}{3}$.

This' is due to the fact that the intensity $I$ of photoelectrons depends also on $\Theta$. In case 2 it is larger for $\Theta= \pm 90^{\circ}$ than for $\Theta=0^{\circ}, 180^{\circ}$. If $D_{s}=0$, i.e. the 


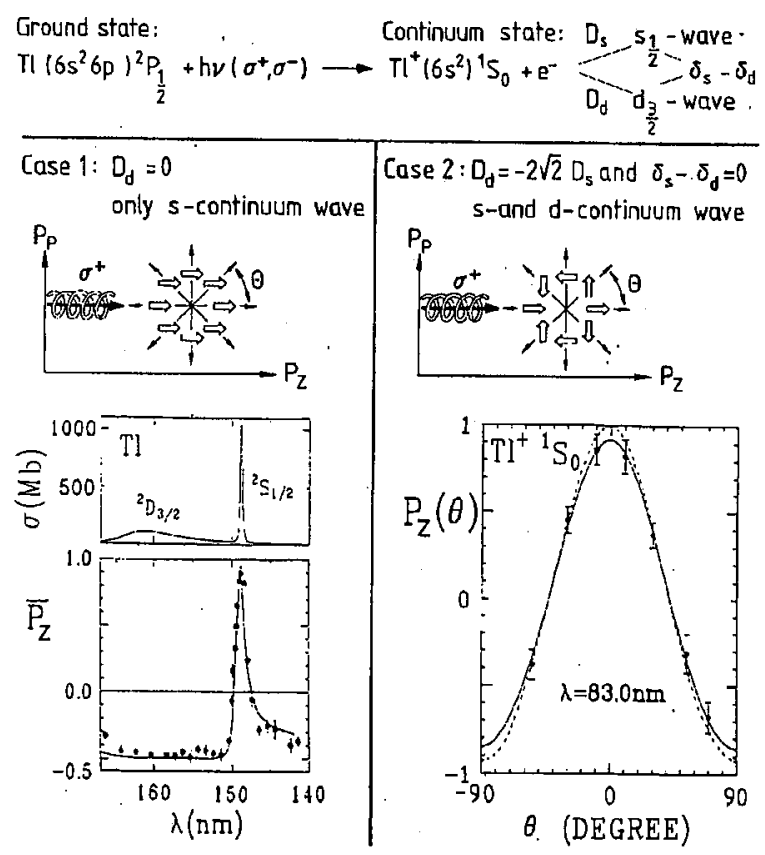

Fig. 7. Top: reaction equation describing the photoionization of the $\mathrm{Tl} 6 p$ level with $\sigma$-radiation. Bottom: the two cases for which $\boldsymbol{P}=1$ can be achieved simultaneously for all $\Theta$. Left: case 1, experimental result from Müller et al., Ref. [28]. The experimental $\bar{P}_{z}$ data (dots with error bars) are compared with a calculation of Cherepkov (solid line). Right: case 2, experimental result from Böwering et al., Ref. [28].

transition occurs into a pure $d$-state, $\bar{P}_{z}=-0.5$. In general $I$ and both $|\boldsymbol{P}|$ and the orientation of $\boldsymbol{P}$ depend on $\Theta$ (see Ref. [22]).

\subsection{Probing magnetic structure with circularly polarized light: $C M X D$, $S P E X A F S$ in absorption and MCD in photoemission}

The techniques for probing magnetic structure displayed in Fig. 8 use circularly polarized radiation to produce a spin polarized electron by optical orientation in a transition from a $p$-level, i.e. exactly as in the photoionization of $\mathrm{Tl}$.

In circular magnetic X-ray dichroism (CMXD) [11], the transitions occur into the unoccupied part of the exchange split valence bands only some $\mathrm{eV}$ above the Fermi level. Since the spin-up and spin-down density of states are different and since left and right circularly polarized radiation yields spin polarized electrons with opposite signs the absorption of circularly polarized radiation is helicity-dependent. From the helicity dependence the local magnetic moments $\mu$ can be deduced

$$
\mu \propto \frac{\alpha^{\uparrow \uparrow}-\alpha^{\uparrow \downarrow}}{\bar{P}_{z}}
$$

with $\alpha^{\uparrow \uparrow}$ and $\alpha^{\uparrow \downarrow}$ - the attenuation coefficients $\alpha$ for photon spin parallel $(\alpha \uparrow \uparrow)$ and antiparallel to the magnetization $M$ of the sample. $\bar{P}_{z}$ is -0.5 for transitions 


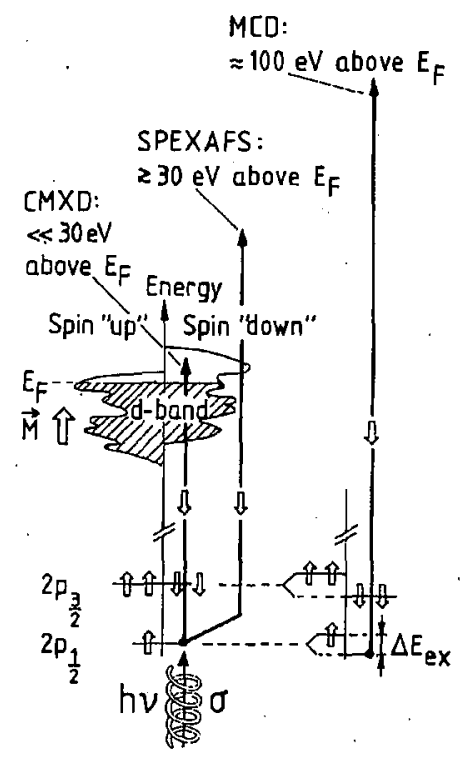

Fig. 8. Probing magnetic properties of matter with circularly polarized X-rays. Explanations are given in the text.

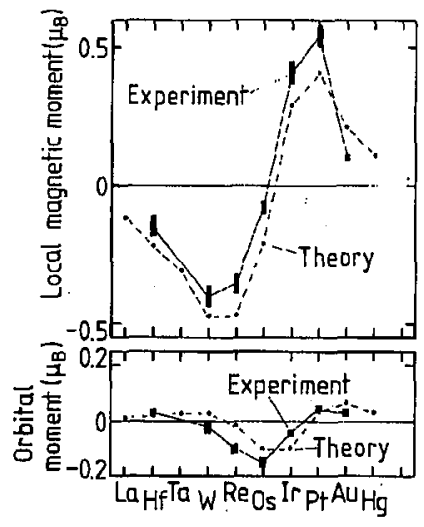

Fig. 9. Local magnetic moments for 5 d-impurities in $\mathrm{Fe}$ determined from CMXD spectra. Top: local magnetic moments. Bottom: estimation of orbital moments using the different absorption behaviour for the $L_{3}$ - and $L_{2}$-edges (experimental data from $[11,29,31]$, theoretical values from [32]).

from the $p_{1 / 2}$ level into pure $d$-states and +0.25 for the corresponding transitions from the $p_{3 / 2}$ level.

Local magnetic moments for a number of heavy impurities in iron are given in the upper part of Fig. 9. When going from Os to Ir a change between the ferromagnetic and antiferromagnetic coupling to the $\mathrm{Fe}$ lattice is observed $[11,29]$. Presently different groups [30] are working on a separation of these mag- 
netic moments into their spin and orbital parts, which yields a deeper insight into the interplay of Coulomb and spin-orbit interactions, hybridization and crystal fields [30] and is also required for a deeper understanding of magnetism.

The experimental [31] and theoretical [32] values of the orbital moments for impurities in iron are given in the lower part of Fig. 9. Orbital moments turn out to contribute considerably to the total magnetic moment and can become even dominant for Os, i.e. close to the point of change between ferromagnetic and antiferromagnetic coupling.

Spin polarized extended X-ray absorption fine structure [12] makes use of spin polarized electrons with kinetic energies larger than about $30 \mathrm{eV}$. Scattering processes with neighbouring atoms depend on the electron spin polarization and thus also on the handedness of the radiation. Information is gained about the magnetic short range order [12].

In the magnetic circular dichroism photoemission experiments described below [7] the photoelectron energies are chosen some $100 \mathrm{eV}$ above the Fermi level. Dependencies of the photoelectron intensity on the handedness of the radiation can be expected, if - in addition to the spin-orbit splitting - an exchange splitting exists, which separates the levels for spin-up and spin-down electrons energetically. Due to the limited photoelectron range MCD in photoemission is a tool for the study of surface magnetism.

An example for MCD in the photoemission from core levels of iron [7] is displayed in Fig. 10. The sample magnetization $M$ is parallel to the surface and

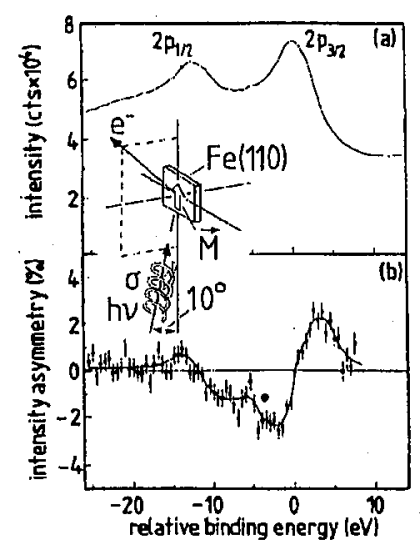

Fig. 10. MCD in the photoemission from the $2 p$ core levels of iron. Inset: experimental geometry. Top: total photoelectron intensity $I$. Bottom: asymmetry given by $\frac{I\left(\sigma^{+}\right)-I\left(\sigma^{-}\right)}{I\left(\sigma^{+}\right)+I\left(\sigma^{-}\right)}$ (after Baumgarten et al., Ref. [7]).

the light incidence occurs at grazing angle of $10^{\circ}$ with respect to the surface in order to have a large component of $M$ aligned with the direction of light propagation. Switching the light helicity from $\sigma^{+}$to $\sigma^{-}$results in a change of the photoelectron intensity $I$ from $I\left(\sigma^{+}\right)$to $I\left(\sigma^{-}\right)$and yields the asymmetry $\frac{I\left(\sigma^{+}\right)-I\left(\sigma^{-}\right)}{I\left(\sigma^{+}\right)+I\left(\sigma^{-}\right)}$in the 
lower part of Fig. 10. Reversing of the sample magnetization $M$ results in the same asymmetries.

Further results [33] show that MCD in photoemission can even be observed if $M$ is normal to the direction of light propagation due to final state selection effects of angle-resolved photoemission.

\subsection{Spin-, angle- and energy resolved photoemission from non-magnetic solids and adsorbate layers}

Spin-, angle-, and energy resolved photoemission with circularly polarized radiation from non-magnetic matter has in the past several times been applied to the ideal case of solid surfaces [15] and the weakly bound almost free atom-like rare gas adsorbates $[15,34]$.

In the last years a lot of work has been devoted to metal adsorbate layers and intermixed systems on different surfaces [35-44]. The studies include photoemission from $d$-core levels $[35,36]$ which can be described like free atom photoionization, epitaxial layer systems for different coverages [37, 39, 41], systems which interact more or less strongly [37-42] with the underlying substrate as well as intermixed $[42,43]$ and magnetic systems [44]. Some of these experiments are described. All results were obtained for the highly symmetrical setup of normal radiation incidence and normal electron emission.

For the photoemission from the $d$-core levels of a thick unordered thallium layer on Pt the photoemission spectrum in Fig. 11 has been obtained [36]. Due to

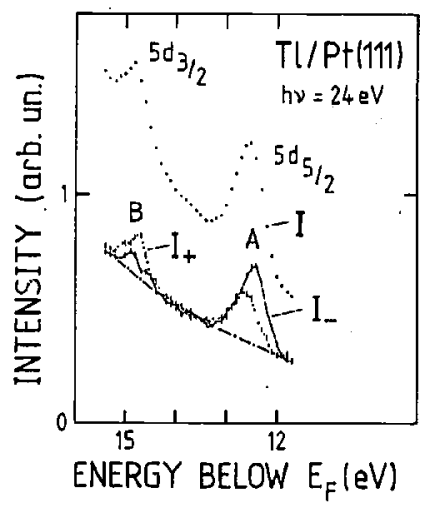

Fig. 11. Spin resolved spectrum for the photoemission from a thallium adsorbate layer on $\operatorname{Pt}(111)$ (from Ref. [36]).

a spin-orbit splitting of $2.3 \mathrm{eV}$ into a $d_{3 / 2}$ and a $d_{5 / 2}$ level two peaks are visible. Spin analysis yields spin polarization values of about $+50 \%$ and $-28 \%$ (with the background subtraction indicated by the dash dotted line below the peaks) for the photoemission from these two levels as can be seen from the separation of the spectrum into its parts $I_{+}$with spin parallel and $I_{-}$with spin antiparallel to the photon spin. These are almost exactly the values which are expected in a free atom 
photoionization experiment for the $5 d$ levels of $\mathrm{Tl}$ (if spin-orbit interaction of the final states is neglected [36]). In addition, a crystal field splitting for the $d_{5 / 2}$ level of about $0.2 \mathrm{eV}$ is detected.

Spin polarized photoemission with circularly polarized radiation from an 8-layer epitaxial $\mathrm{Ag}$ adsorbate on $\mathrm{Pt}(111)$ yields photoemission spectra like the one in Fig. 12 which are identical with those of $\mathrm{Ag}(111)$. Spin analysis reveals 4

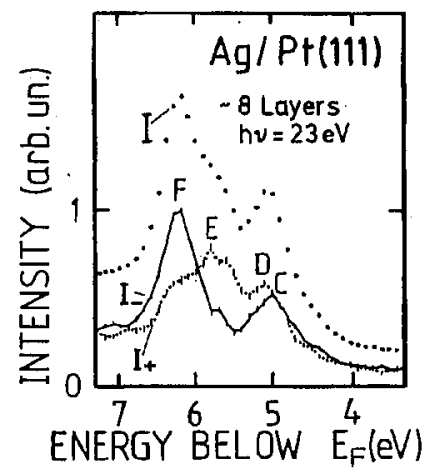

Fig. 12. Spin resolved spectrum for the photoemission from a thick epitaxial Ag-layer on Pt(111) (from Ref. [37]).

peaks $C, D, E, F$ with the spin polarization sign sequence minus-plus-plus-minus. They are due to the transitions from the valence bands of Ag. Compared to the photoionization from the $d$-levels of thallium we have now a large crystal field splitting due to $\mathrm{Ag}-\mathrm{Ag}$ interaction in combination with a by far smaller spin-orbit splitting, which results in the displayed spectrum with 4 peaks. Spin-orbit interaction separates the valence band states into wave functions of $\Lambda_{45}^{3}$ and $\Lambda_{6}^{3}$-symmetry, which yield even completely spin polarized electrons for transitions with circularly polarized radiation into states with symmetry $\Lambda_{6}^{1}$ [37].

The photoemission from $\mathrm{Ag}$ adsorbate layers of different coverages and on different substrates has been studied [37, 40] in the same manner as for $\mathrm{Ag}(111)$ $[37,38]$ and yields almost always with up to now only one exception a photoemission spectrum with 4 peaks and the spin polarization sign sequence minus-plusplus-minus as in Fig. 12. This indicates that in all of these cases Ag-d-electron overlap is present in combination with spin-orbit splitting. The exception is a study of submonolayer $\mathrm{Ag}$ adsorbates on the (111) surface of silicon. Room temperature adsorption of 0.5 monolayers of $\mathrm{Ag}$ results in an adsorbate with $(1 \times 1)$ LEED pattern and the photoemission spectrum with the four Ag peaks, which show the presence of $\mathrm{Ag}-\mathrm{Ag}$ interaction (left part of Fig. 13). Annealing of the sample at $500^{\circ} \mathrm{C}$ transfers the surface into a $\sqrt{3} \times \sqrt{3} R 30^{\circ}$ structure and alters the photoemission spectrum in such a manner that spin polarization analysis reveals only the spin-orbit split peak (see Fig. 13, right) as in the photoionization of $\mathrm{Tl}$ in Fig. 11. No indication for a presence of $\mathrm{Ag}-\mathrm{Ag}$ interaction is found. The (mainly geometric) structure of this adsorbate system is still object of a long on-going 

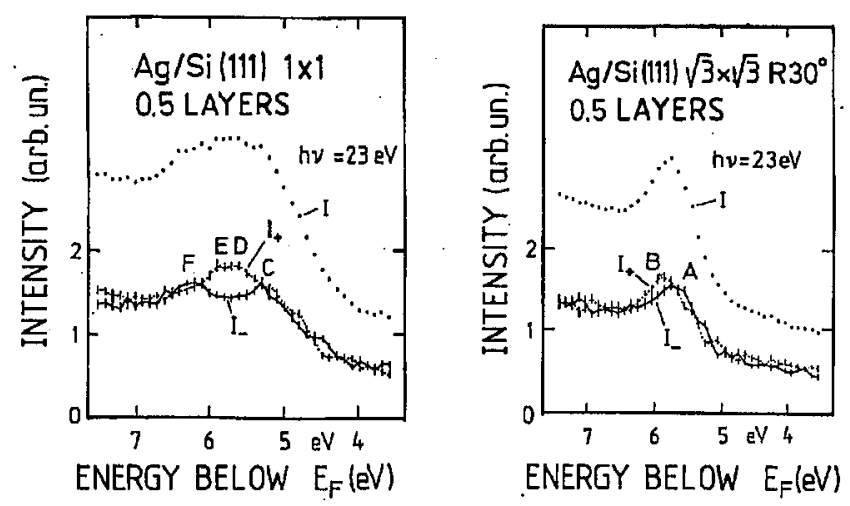

Fig. 13. Detection of Ag valence orbital overlap for Ag adsorbates on $\mathrm{Si}(111)$ by means of spin resolved photoemission (from Vogt et al., Ref. [40]).

debate in literature (several references are given in [40]). Structure models which require $\mathrm{Ag}-\mathrm{Ag}$ interaction can clearly be excluded by the described study.

An understanding of symmetry properties of surface and interface states requires not only adsorbate systems which yield reproducible experimental results; it should also be ideal enough to be treatable theoretically. Au on Pt(111) can be grown epitaxially in a manner that the Au atoms are on lattice sites which are a direct continuation of the Pt lattice [45]. An experimental result for this system is given in the left part of Fig. 14. Spin analysis reveals several peaks which can be

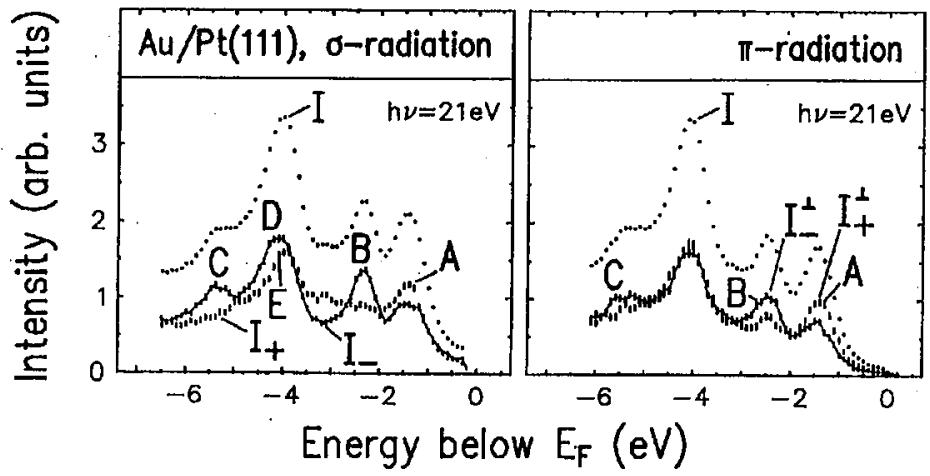

Fig. 14. The surface and interface electronic structure for a Au-monolayer on Pt(111) studied with spin resolved photoemission. Left: photoemission with circularly polarized radiation. Right: photoemission with linearly polarized light (from Ref. [41]). $I_{+}^{\perp}=$ $\frac{I}{2}\left(1+P_{\perp}\right), I_{-}^{\perp}=\frac{I}{2}\left(1-P_{\perp}\right)$

attributed to corresponding structures in a calculation of Tamura and Feder [45]. The spectrum consists of contributions from Pt-surface emission with symmetry $\Lambda_{45}^{3}$ [46] (peak $A$ ), a Au-induced surface state on $\mathrm{Pt}(111)$, which is located in the $A u$ layer and the underlying first Pt layer $[41,45]$ (peak $B$ ), states which are only 
located in the Au adsorbate layer (peak $C$ ) and bulk states of the Pt-substrate (peak $D$ ).

The data contribute also to an understanding of a spin polarization effect with linearly polarized light in the normal photoemission from threefold surfaces [45-47]. Spin polarized electrons of this type are not mentioned in this work up to now, since they occur in photoemission neither with circularly polarized nor with unpolarized light. They require also the spin-orbit interaction but they have no analogy in the photoionization of free atoms or the photoemission in 3-dimensional solids. They seem to be generated only in the photoemission from surfaces and interfaces and are predicted to require electronic states of the symmetry type $\Lambda_{45}^{3}$ [47]. An additional interesting property is the fact that the spin polarization is sensitive to the geometrical structure of surfaces and adsorbate layers [45]. A photoemission spectrum obtained with linearly polarized radiation for the Au monolayer on $\mathrm{Pt}(111)$ is given in the right part of Fig. 12. Pronounced spin polarization $\boldsymbol{P}$ perpendicular to the light direction (component $P_{\perp}, \boldsymbol{P}$ rotates by $3 \phi$, if the sample is rotated by $\phi$ about the surface normal) is found in three of the four peaks. A comparison with the corresponding spectrum for $\sigma$ light (Fig. 14, left) shows that spin polarization is indeed only obtained for photoemission from those states which have both surface or interface location and symmetry $\Lambda_{45}^{3}$.

\subsection{Spin polarized Auger electrons after core hole generation with circularly polarized light}

The photoemission process with circularly polarized radiation produces in general spin polarized photoelectrons due to optical orientation. It generates, however, as illustrated for the case of a Rb metal layer in Fig. 15, also a hole which can decay via emission of an Auger electron. Since the emitted electron is spin polar-

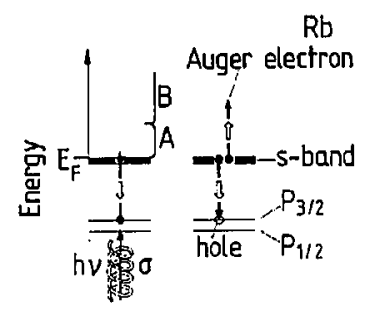

Fig. 15. Spin polarized Auger electrons from Rb layers. Left: generation of a "spin polarized hole" with $\sigma$-radiation. Right: decay of the hole and emission of a spin-polarized Auger electron. $A, B$ : see text.

ized one expects also a spin polarization for the hole left behind and the question arises whether and in which manner spin polarization is transferred from the hole state onto the Auger electron in the Auger decay.

The question has been addressed to alkali layers [19]. A high degree of spin polarization was found for the emitted Auger electrons. Results for the Rb metal layers are given in Fig. 16. A change of sign is observed for the spin polarization 


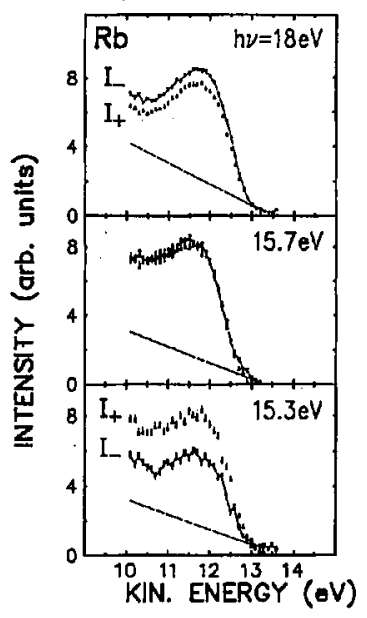

Fig. 16. Spin polarized Auger electrons from Rb layers, experimental result (from Stoppmanns et al., Ref. [19]).

at a photon energy of $15.7 \mathrm{eV}$. This can be explained by the fact that the density of states above the Fermi level changes from dominantly $s$-like directly above $E_{\mathrm{F}}$ (indicated by $A$ in Fig. 15) to dominantly $d$-like at higher energies ( $B$ in Fig. 15). Taking this into account the results can qualitatively be explained in a simple model. In step 1 a polarized $p_{3 / 2}$-hole is generated. Its spin polarization sign depends on whether the electron is transferred into $s$ - or $d$-states. In step 2 the Auger decay is regarded in an atom-like model. Momentum conservation demands then that the spin polarization vector of the emitted Auger electron is opposite to the spin polarization vector of the electron emitted in step 1. Since the spin polarization of this electron depends on the symmetry of the final states also for energies between $E_{\mathrm{F}}$ and the vacuum level, spin polarized Auger electron spectroscopy can be used to deduce symmetry properties of states in this energy range and opens more generally a new method of studying excitations and unoccupied electronic states.

\section{Summary and outlook}

$\mathrm{X}$-ray absorption is the first step in all experiments with circularly polarized radiation. It can be studied by measuring the intensity transmitted through a sample. It can be different for right and left-handed circularly polarized radiation if the sample is magnetic or chiral. X-ray absorption of circularly polarized radiation results also in the emission of spin-polarized photoelectrons. Not only do they carry information about magnetic properties but also about the fundamental electronic properties of matter. Considerations of X-ray absorption are also necessary if the beamlines for experiments with $\sigma$-radiation are designed.

Future experiments with $\sigma$-radiation will certainly profit considerably from wiggler and undulator sources which are presently put into operation in several 
synchrotron radiation laboratories and which yield higher fluxes of $\sigma$-radiation especially at higher photon energies. Together with further properties such as the tunability of the radiation wavelength and the degree of circular polarization, the ability of a fast change of the radiation helicity and a time structure which allows time resolved studies, one can expect considerable progress. Examples are photoemission and Auger processes involving core levels and even aligned and oriented molecules, time resolved studies of CD (in absorption and fluorescence) from chiral matter (in order to study the kinetics of structure changes), circular differential X-ray microscopy, MCD from large molecules or impurities and defects in solids, and light scattering from molecules.

\section{Acknowledgments}

I would like to thank Prof. Dr. U. Heinzmann for his continuous support and fruitful discussions. Several of the above-described experiments were done within the scope of projects of his group. They could not have been performed without the experimental collaboration with R. David, N. Irmer, B. Kessler, N. Müller, P. Stoppmanns and B. Vogt, Universität Bielefeld (spin resolved photoemission and Auger experiments), U. Kleineberg, A. Kloidt, H.J. Stock, Universität Bielefeld, M.K. Krumrey, P. Müller, F. Scholze, PTB Berlin and K.F. Heidemann, Carl Zeiss Oberkochen (multilayer optics). Thanks are also due to the BESSY staff and colleagues of the Fritz-Haber-Institut der MPG. Cooperation with several further colleagues whose data were shown in the lecture is gratefully acknowledged: N. Böwering, R. Feder, J. Kirschner, M. Müller, C.M. Schneider, G. Schütz, E. Spiller and E. Tamura. Several of the experiments were financially supported by BMFT and DFG.

\section{References}

[1] A.G. Michette, Optical Systems for Soft X-Rays, Plenum, New York 1986.

[2] B.L. Henke, P. Lee, T.J. Tanaka, R.L. Shimabukuru, B.K. Fujikawa, AIP Proc. 75, 340 (1981); At. Data Nucl. Data Tables 27, 1 (1982); B.L. Henke, J.C. Davis, E.M. Gullikson, R.C. Perera, Preliminary report on the updating of the tables, Lawrence Berkeley Laboratory, Berkeley, California 94720, 1994.

[3] M. Born, E. Wolf, Principles of Optics, 6th ed., Pergamon Press, Oxford 1987.

[4] For a review on multilayer soft X-ray optics see E. Spiller, in: Thin Films for Optical Coatings, Springer Series Topics in Applied Physics, to be published.

[5] U. Heinzmann, J. Phys. B, At. Mol. Phys. 13, 4353 (1980).

[6] F. Schäfers, W. Peatman, A. Eyers, Ch. Heckenkamp, G. Schönhense, U. Heinzmann, Rev. Sci. Instrum. 57, 1032 (1986).

[7] L. Baumgarten, C.M. Schneider, H. Petersen, F. Schäfers, J. Kirschner, Phys. Rev. Lett. 65, 492 (1990).

[8] C.T. Chen, F. Sette, Y. Ma, S. Modesti, Phys. Rev. B 42, 7262 (1990).

[9] J.B. Kortwright, J.H. Underwood, Nucl. Instrum. Methods Phys. Res. A 291, 272 (1990); S. Di Fonzo, W. Jark, Rev. Sci. Instrum. 63, 1375 (1992); M. Yamamoto, M. Yanagihara, H. Nomura, K. Mayama, H. Kimura, Rev. Sci. Instrum. 63, 1510 (1992). 
[10] T. Ishikawa, K. Hirano, K. Kanzaki, S. Kikuta, Rev. Sci. Instrum. 63, 1098 (1992).

[11] G. Schütz, W. Wagner, W. Wilhelm, P. Kienle, R. Zeller, R. Frahm, G. Materlik, Phys. Rev. Lett. 58, 737 (1987); G. Schütz, Phys. Bl. 46, 475 (1990).

[12] G. Schütz, R. Frahm, P. Mautner, R. Wienke, W. Wagner, W. Wilhelm, P. Kienle, Phys. Rev. Lett. 62, 2620 (1989).

[13] See several contributions in Rev. Sci. Instrum. 63 (1992): J. Pflüger, p. 295, S. Yamamoto, T. Shioya, M. Hara, H. Kitamura, X.W. Zhang, T. Mochizuki, H. Sugiyama, M. Ando, p. 400, S. Onuki et al., p. 173, Y. Kagoshima, S. Muto, T. Miyahara, T. Koide, S. Yamamoto, H. Kitamura, p. 1289, J. Bahrdt, A. Gaupp, W. Gudat, M. Mast, K. Molter, W.B. Beatman, M. Scheer, Th. Schroeter, Ch. Wang, p. 339 , P. Ellaume, p. 321.

[14] U. Heinzmann, in: Fundamental Processes in Atomic Collision Physics, Eds. H. Kleinpoppen, J.S. Briggs, H.O. Lutz, Plenum, New York 1985, p. 269.

[15] U. Heinzmann, Phys. Scr. Vol. T 17, 77 (1987), and in: Photoemission and Absorption Spectroscopy of Solids and Interfaces with Synchrotron Radiation, Eds. M. Campagna, R. Rosei, North-Holland, Amsterdam 1990, p. 469.

[16] F. Meier, D. Pescia, in: Optical Orientation, Eds. F. Meier, B.P. Zakharchenya, North-Holland, Amsterdam 1984, Ch. 7.

[17] J. Kirschner, Polarized Electrons at Surfaces, Springer, Berlin 1985.

[18] Polarized Electrons in Surface Physics, Ed. R. Feder, World Scientific, Singapore 1985.

[19] P. Stoppmanns, B. Schmiedeskamp, B. Vogt, N. Müller, U. Heinzmann, Phys. Scr. Vol. T 41, 190 (1992).

[20] E. Charney, The Molecular Basis of Optical Activity: Optical Rotary Dispersion and Circular Dichroism, Wiley, New York 1979; M. Carnell, S.D. Peyerimhoff, A. Breest, U.H. Gödderz, P. Ochmann, J. Hormes, Chem. Phys. Lett. 180, 427 (1991).

[21] G. Schönhense, Phys. Scr. Vol. T 31, 255 (1990).

[22] U. Heinzmann, J. Phys. B, At. Mol. Phys. 13, 4367 (1980).

[23] N.A. Cherepkov, J. Phys. B, At. Mol. Phys. 12, 1279 (1979); K.N. Huang, Phys. Rev. A 22, 223 (1980); H. Klar, J. Phys. B, At. Mol. Phys. 13, 3117 (1980).

[24] J. Kessler, Polarized Electrons, 2nd ed. Springer, Berlin 1985.

[25] U. Fano, Phys. Rev. 178, 131 (1969) and Phys. Rev. 184, 250 (1969).

[26] U. Heinzmann, J. Kessler, J. Lorenz, Phys. Rev. Lett. 25, 1325 (1970).

[27] Ch. Heckenkamp, F. Schäfers, G. Schönhense, U. Heinzmann, Phys. Rev. Lett. 52, 421 (1984).

[28] N. Böwering, M. Salzmann, M. Müller, H.W. Klausing, U. Heinzmann, Phys. Scr. 41, 429 (1990); M. Müller, N. Böwering, A. Svensson, U. Heinzmann, J. Phys. B 23, 2267 (1990).

[29] R. Wienke, G. Schütz, H. Ebert, J. Appl. Phys. 69, 6147 (1991).

[30] B.T. Thole, P. Carra, F. Sette, G. van der Laan, Phys. Rev. Lett. 68, 1943 (1992) and references therein.

[31] G. Schütz, private communication.

[32] H. Ebert, R. Zeller, B. Drittler, P. Dederichs, J. Appl. Phys. 67, 4576 (1990).

[33] C.M. Schneider, D. Venus, J. Kirschner, Phys. Rev. B 45, 5041 (1992). 
[34] U. Heinzmann, G. Schönhense, Ch. 11 of Ref. 18 in G. Schönhense, Appl. Phys. A 41,39 (1986).

[35] B. Vogt, B. Schmiedeskamp, U. Heinzmann, Vacuum 41, 1118 (1990).

[36] B. Schmiedeskamp, B. Vogt, U. Heinzmann, Z. Phys. B 90, 197 (1993).

[37] B. Schmiedeskamp, B. Kessler, B. Vogt, U. Heinzmann, Surf. Sci. 223, 465 (1989).

[38] E. Tamura, R. Feder, B. Vogt, B. Schmiedeskamp, U. Heinzmann, Z. Phys. B 77, 129 (1989).

[39] B. Vogt, B. Schmiedeskamp, U. Heinzmann, Z. Phys. B 80, 359 (1990).

[40] B. Vogt, B. Schmiedeskamp, U. Heinzmann, Phys. Rev. B 42, 9267 (1990).

[41] B. Schmiedeskamp, N. Irmer, P. Stoppmanns, B. Vogt, U. Heinzmann, Europhys. Lett. 20, 657 (1992).

[42] B. Vogt, P. Stoppmanns, B. Schmiedeskamp, U. Heinzmann, Appl. Phys. A 52, 323 (1991).

[43] C.M. Schneider, G.S. Sohal, P. Schuster, J. Kirschner, Vacuum 41, 511 (1990); C.M. Schneider, J.J. de Miguel, P. Bressler, P. Schuster, R. Miranda, J. Kirschner, J. Electron Spectrosc. Relat. Phenom. 51, 263 (1990).

[44] C.M. Schneider, P. Schuster, M.S. Hammond, J. Kirschner, Europhys. Lett. 16, 689 (1991).

[45] P. Stoppmanns, B. Heidemann, N. Irmer, N. Müller, B. Vogt, B. Schmiedeskamp, U. Heinzmann, E. Tamura, R. Feder, Phys. Rev. Lett. 66, 2645 (1991).

[46] B. Schmiedeskamp, B. Vogt, U. Heinzmann, Phys. Rev. Lett. 60, 651 (1988).

[47] E. Tamura, W. Piepke, R. Feder, Phys. Rev. Lett. 59, 934 (1987).

[48] B. Schmiedeskamp, A. Kloidt, H.J. Stock, U. Kleineberg, T. Döhring, M. Pröpper, S. Rahn, K. Hilgers, B. Heidemann, T. Tappe, U. Heinzmann, M.K. Krumrey, P. Müller, F. Scholze, K.F. Heidemann, Opt. Eng. 33, 1314 (1994); H.J. Stock U. Kleineberg, A. Kloidt, B. Schmiedeskamp, U. Heinzmann, M. Krumrey, P. Müller, F. Scholze, Appl. Phys. Lett. 63, 2207 (1993). 